\title{
Novel Method of Contactless Sensing of Mechanical Quantities
}

\author{
René Hart’anský1 ${ }^{1}$, Martin Mierka ${ }^{1}$, Mikuláš Bittera ${ }^{1}$, Jozef Hallon ${ }^{1}$, Ján Halgoš ${ }^{1}$, Jaroslav Hricko², \\ Robert Andok ${ }^{2}$, Michal Rafaj ${ }^{3}$ \\ ${ }^{1}$ Institute of Electrical Engineering, Department of Measurement, Faculty of Electrical Engineering and Information \\ Technology, Slovak University of Technology, Ilkovičova, No. 3, 81219 Bratislava, Slovakia, xmierka@stuba.sk \\ ${ }^{2}$ Institute of Informatics, Slovak Academy of Science, Dúbravská cesta, No. 9, 84507 Bratislava, Slovakia \\ ${ }^{3}$ RMC ltd., Trenčianska, No. 863/66a, 01851 Nová Dubnica, Slovakia
}

\begin{abstract}
This article addresses the method of sensing mechanical quantities, in particular force and pressure, without the electrical connection of the sensing element and the electronics. The information about the mechanical quantity is transmitted only by evaluating the changes in the electromagnetic field created around the sensor. The sensor is designed on the basis of a flexible micro-electro-mechanical element (MEMS), the resonance of which carries the information about the measured quantity.
\end{abstract}

Keywords: Measurement of physical quantities, MEMS, electromagnetic field, reflection parameters, S parameters.

\section{INTRODUCTION}

General trends in the development of technical elements and equipment are characterized by the reduction of size and weight requirements, increase of functional parameters and performance, as well as by saving energy and use of expensive materials. Advances in the development of technologies, materials, but mainly sensor and informationmanagement systems in recent years have allowed the emergence of new directions stimulated by new applications. New applications in micro- and optoelectronics, medicine, biology, and elsewhere envisage development of micro-electro-mechanical components and devices (MEMS) together with the development of corresponding manufacturing micro-technologies, materials, principles of sensing, measuring and processing signals, and control. Besides the miniaturization of dimensions, MEMS materials and technologies have allowed a substantial increase in sensing quality as well as in resistance of the mechanical sensors of quantity (force, pressure, position change, etc.) for the use in automation, modern robotics [1], medicine [2] and so on, and of chemical parameters in various sectors, including that of space research and public safety.

In the case of MEMS it is obvious that conventional constructions consisting of discrete elements are not able to accomplish the given requirements. A solution is in compact compliant structures with elastically tractable connections of individual devices, while their miniaturization can be attained only by using the microsystems (MEMS) technology and other highly accurate production methods. Such structures can be used as mechanisms of micropositioning devices, actuators and micro-effectors, as various tools for accurate micromanipulation in a clean environment, in optoelectronics, microsurgery, etc. A wide group of MEMS devices represent activators and transducers (of e.g., force/moment of force, pressure, speed, acceleration, flow, etc.) [3], [4]. In the solution of such mechanical structures, specific approaches to design, kinematic, force and deformation analysis, are applied.

Within the automation of technological processes and miniaturization, technical equipment is considered one of the main issues in solving the problem of signal and energy transmission. In this area there is a shift from electrical conductors to wireless technologies. This evolution in communication and energy transfer is required especially in the areas where it is not possible to use electric wires. As an example let us mention the extensive research at Japanese universities (Nippon Institute of Technology, Kyushu Institute of Technology, and others) where the researchers specialize in application use of flexible structures and wireless transmission of energy in medical applications (microcapsule - a wireless implementation where a camera together with microcapsule in a human body is driven by electromagnetic pulses [5], or various catheters and microboots controlled wirelessly [6], [7]).

Multinational corporations such as ABB, Honeywell Process Solutions and others, are considered to be the leaders in $R \& D$ in the area of wireless sensors used in 
industry. From the global market development reports a stable growth is expected (more than $20 \%$ until 2021) as industry replaces cable sensors with their wireless equivalents because it enables industrial areas to achieve better expense and energy efficiency.

In the H 2020 program, over 200 projects are solved that fall within the sensors problematics (or their direct use). Some of these projects focus mainly on contactless/wireless transmission of energy, especially when using IoT devices. Another problem solved within these European projects are different sensor networks and wireless communication in medicine (surgical implants), respectively. Continuous trend to focus on micro-mechatronic systems confirms an intense research at many leading universities as well as research institutes in the world (in Europe being supported explicitly in the specified direction).

\section{SUBJECT \& METHODS}

The basic idea of contactless sensing of a physical quantity is in using the MEMS in the form of a converter of acting mechanical force, pressure, etc., to such a quantity or process that will affect the properties of the surrounding electromagnetic field (EMF). Ultimately, a change in EMF parameters will carry the information about the acting mechanical quantity. The method described below does not use the known RF MEMS or RFID principles, thus in this chapter we will try to get closer to the physical principles of measurement and transmission of information.

\subsection{Mutual impedance of parallel wires}

In the theory of antennas, a mutual task of the influence of wiring structures that are in their immediate proximity is often mentioned. For the generation of EMF by using these structures, the case when they exhibit the same or very close dimensions is of particular interest. However, the authors in [10] began to investigate the structures significantly differing in their size. The investigated model can be seen in Fig.1. The wire - radiator had its length $l_{l}=0.462 \mathrm{~m}$ and diameter $a=1.8 \mathrm{~mm}$. In a distance of $0.780 \mathrm{~m}$ another wire was placed with its length varying (while the diameter remained constant $-1.8 \mathrm{~mm}$ ).

In [10] the real part of the input impedance of the radiator was examined in dependence to frequency. Three model situations were created: without "metallic wire", with "metallic wire" of $l_{2}=0.51 \mathrm{~m}$, and with "metallic wire" of $l_{2}=0.61 \mathrm{~m}$, respectively. The result of numerical simulation is shown in Fig. 2.

We may see that the input impedance of the radiator is determined not only by the existence of the "metallic wire" in its immediate vicinity but also by its length. It means that from the frequency dependence of the radiator's input impedance we can calculate the length of the neighboring conductive objects. This effect can be used for measuring the length or distance. However, as we cannot prevent that in the EMF (excited by a radiator) there is only one wire or conductive object present, the neighboring surroundings can, to a significant extent, influence the accuracy of the measurement as well as its realization.

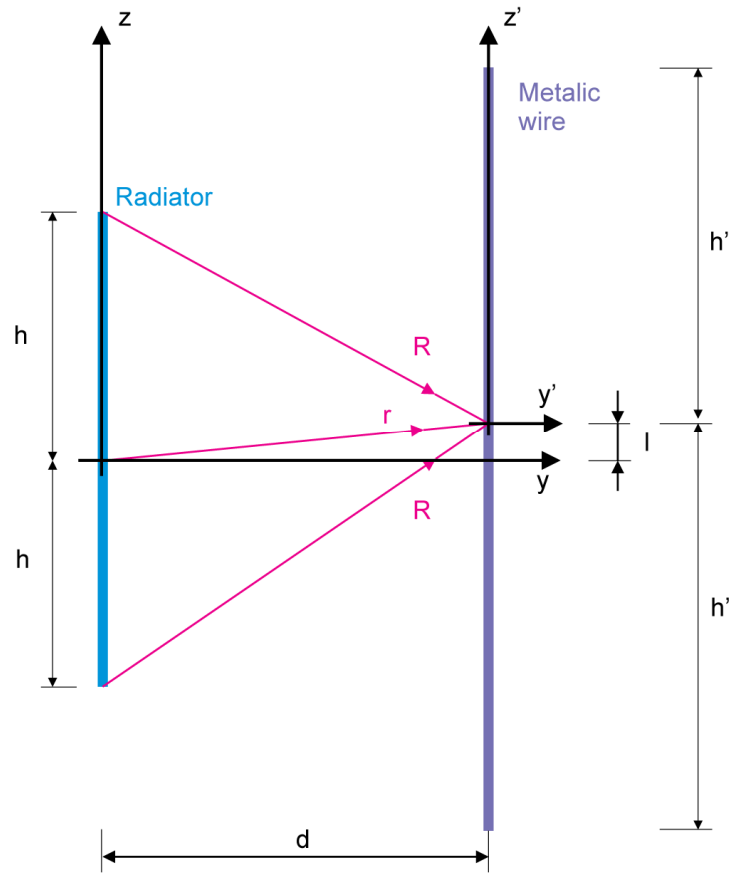

Fig.1. Design of the two wires.

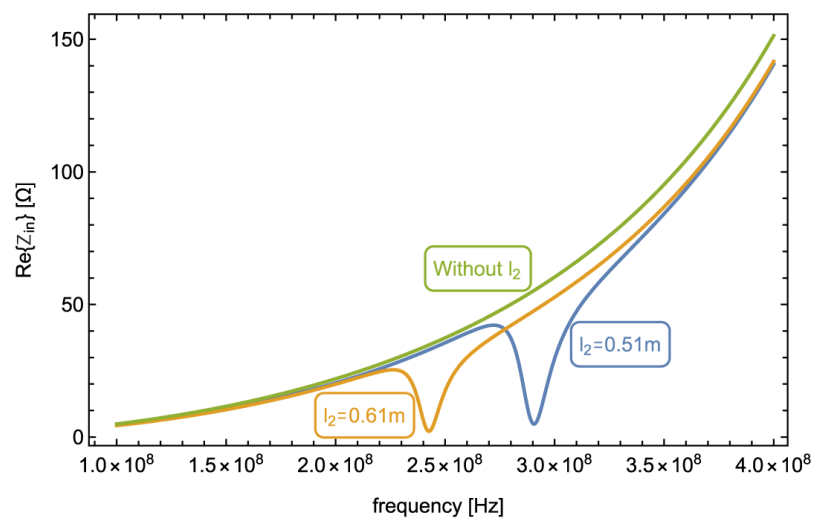

Fig.2. Real part of the radiator input impedance.

\subsection{Mutual impedance of the radiator and the resonance circuit}

In [11] the radiator was replaced by a stripline, Fig.3. The EMF is concentrated into the area between two plates by which its dispersion into the neighborhood is minimized. At the same time the curve overshoot will be increased, as shown in Fig.2. The use of a stripline will thus allow using a rather simple electronic device for data collection and evaluation.

The mechanism of influencing the input impedance of the radiator through a nearby metallic wire consists of the drainage and return of electrical energy from the EMF [11]. This phenomenon is most observable at frequencies which are close to the resonant frequency of the metallic wire. Hence came the idea of replacing the metallic wire with a parallel resonance circuit (PRC). 
If the PRC is a part of the sensor of a mechanical quantity, while its dimensions vary by changing the effect of this quantity, then the resonant frequency of the PRC will carry information about this quantity.

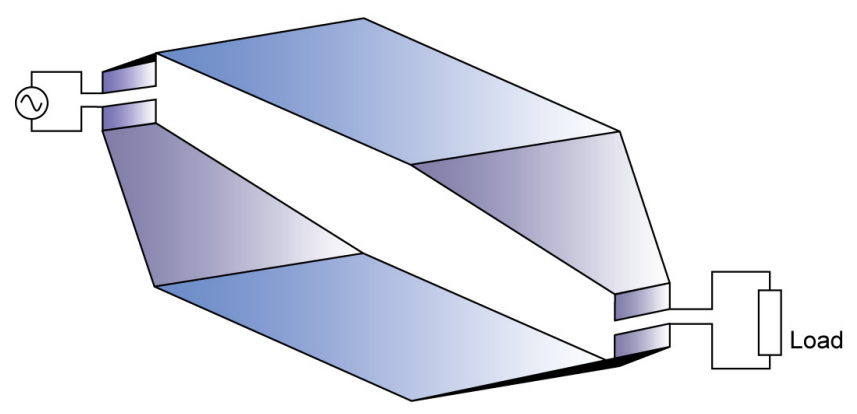

Fig.3. Stripline as a radiator of the EMF.

\subsection{Sensor of physical quantity}

In general, the elastic deformation of a material is the key condition of many force transducers. They depend on the relationship between the acting load $\mathbf{F}$, deflections $\mathbf{u}$ (displacements and rotations), and shape of the deformable part, as well as material properties expressed by the compliance $\mathbf{C}$ or stiffness $\mathbf{K}$, respectively:

$$
\mathbf{u}=\mathbf{C F} \rightarrow \mathbf{F}=\mathbf{K u}
$$

For the proposed novel method of sensing mechanical quantities, the specific requirements are imposed for the design of the force sensor's mechanical part. The functional requirements are similar to common sensors (accuracy in a specific frequency range, compactness, etc.) but especially as a consequence of the PRC integration to the mechanical part, the selected plates should move strongly in parallel (these should represent the capacitor plates). The additional design requirements could be connected with the production technology.

The most well-known shape of the sensor's deformation part is the load cells [13] where the simplest shape is a flexible beam with which it is possible to design multicomponent force sensors [14]. In the design of a suitable flexible body of the sensor it was expected that the general shape of the load cell (Fig.4.a) could be used. Unfortunately, the numerical simulations have shown that the deformation of the moving plate is present and could not be neglected. With the length of the moving plate of $\mathrm{a}_{1}=20 \mathrm{~mm}$, its deformation is up to $160 \mu \mathrm{m}$ (see Fig.4., dependence for the Load cell A). As a result, new shapes of sensor deformable parts have to be designed where the observed deformation is $140 \mu \mathrm{m}$ for design $\mathrm{B}$ and only $85 \mu \mathrm{m}$ for design $\mathrm{C}$ (see Fig.4., Load cell $B$, for the length of $\mathrm{a}_{2}=30 \mathrm{~mm}$, Load cell $\mathrm{C}$, for the length of $\mathrm{a}_{3}=40 \mathrm{~mm}$ ).

After the shape optimization of the proposed deformable part of the sensor, the careful stiffness analysis should be carried out. For the purposes of stiffness modelling, it is assumed that all arisen deformations (deflections) are within the validity of Hook's law. The stiffness of the proposed device is calculated based on the formula for the parallel configuration of the elastic elements [8]:

$$
\mathbf{K}=\sum_{n}{ }^{i} \mathbf{T}_{j} \mathbf{K}_{i}\left({ }^{i} \mathbf{T}_{j}\right)^{T}
$$

where ${ }^{i} \mathbf{T}_{j}$ is transformation matrix between local coordinate systems. The symmetry of the proposed device enables us to calculate only the compliance/stiffness of one curved beam (flexure). Then the results are multiplied by two and applied to the observed point where the external load is present. The curved beam compliance/stiffness matrix is expressed by using Castigliano's theorem where the partial derivatives of the strain energy $U$ are calculated as follows:

$$
U=\frac{1}{2 E} \int_{0}^{\pi}\left(\frac{F_{x}(\phi)^{2}}{A_{k}}+\frac{F_{y}(\phi)^{2}}{A_{k}}+\frac{M_{z}(\phi)^{2}}{I_{z k}}\right) d \phi
$$

where $A_{k}$ and $I_{z k}$ are the area of cross-section and the second moment of the area, respectively. $E$ is Young's modulus of elasticity and $\phi$ is the angle/variable of the function described by the radius of the curved beam. For careful calculations it is necessary to take into account that $A_{k}$ and $I_{z k}$ are functions dependent on the angle $\phi$. Then, the deflection vector $\mathbf{u}$ is calculated as

$$
\mathbf{u}=\frac{\partial U}{\partial \mathbf{F}(\phi)}
$$

After adjusting the results from equation (4) to the form of equation (1), we get the compliance/stiffness matrix of the curved beam that is used in other analyses (full stiffness calculation according to (3), Eigen values, frequency analysis, etc.).

The laboratory physical model of the sensor body has been fabricated by $3 \mathrm{D}$ printing technology. The polyamide with Young's modulus $580 \mathrm{MPa}$ and yield strength $28 \mathrm{MPa}$ was used. As a consequence, nylon is a hygroscopic material, its material properties degrade in time (the strength and stiffness decrease in dependence on the amount of absorbed moisture) and the produced laboratory samples have the desired performance characteristics for a specific short time of a few weeks/months. While decreasing stiffness has a direct impact on the measuring range, nevertheless it is expected that the material of the industrial sensor body will be ceramic or plastic where the influence of the environment has zero effect (e.g., intrinsic silicon). The dimensions of the laboratory model are: width $50 \mathrm{~mm}$, height $11 \mathrm{~mm}$, thickness $5 \mathrm{~mm}$, respectively. As shown in Fig.5., the dependence between an acting load and output displacement is nearly linear. To minimize the sources of measurement errors (e.g., the weight and torque of signal wires transmitting information from the body to the evaluation device) a new method for measuring mechanical quantity has to be invented. 


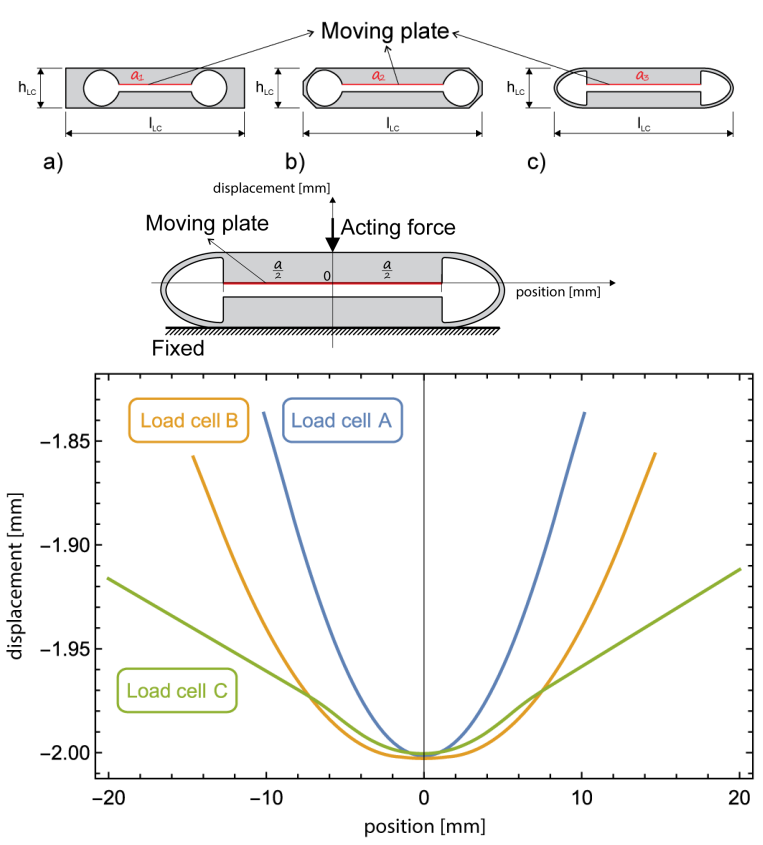

Fig.4. Analyzed shapes of the load cells (top); deformation of the moving plate (down).

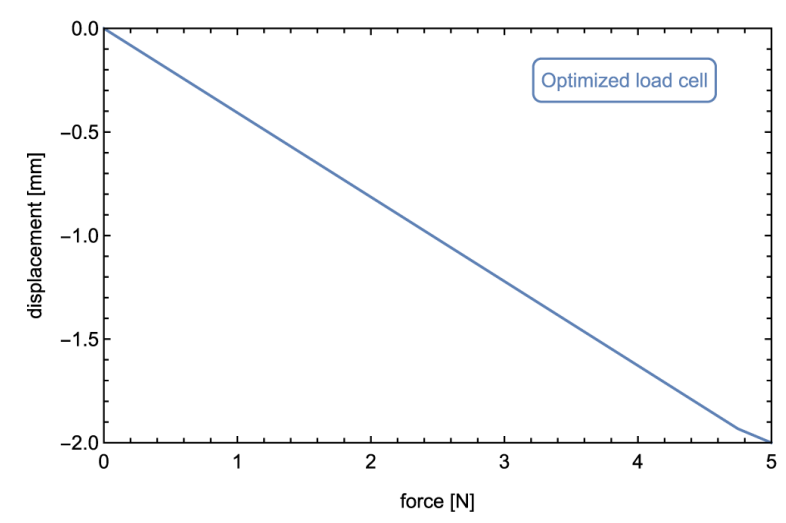

Fig.5. Output characteristic of the proposed sensor body.

\section{Realization of the DeVice for Contactless Sensing OF MECHANICAL QUANTITIES}

Based on the principle of the transfer of information and the principle of the transfer of a mechanical quantity to electrical, which have been mentioned in the previous chapters, a block diagram was proposed [12] using the principle described in Fig.6.

In the voltage-controlled oscillator (VCO) a highfrequency voltage is generated which passes directly through the directional coupler onto the radiator terminals. It could be a stripline or a TEMCell [17] (in Fig.7. the TEMCell is shown). The radiator will generate an electromagnetic field. In the electromagnetic field a mechanical unit (Convertor) with a parallel resonant circuit (PRC) is placed. Due to the mechanical quantity acting to this mechanical unit with the PRC also the distance of the capacitor plates will be changed (see Fig.8.), which causes the change of its capacity and thus change of the resonant frequency of the PRC. The resonant circuit will affect the electromagnetic field created by the radiator. The most significant influence occurs at frequencies close to the resonant frequency of the resonant circuit, causing exhausting of energy of the EMF and its subsequent return (reflection) [15]. The returning wave advances back to the radiator and from there, in the form of a high-frequency voltage it passes through a directional coupler into the control electronics. By comparing the reflected wave and the control voltage $\mathrm{VCO}$, the control electronics calculates the resonant frequency of the PRC, and by that also the value of the non-electrical physical quantity affecting the mechanical component.

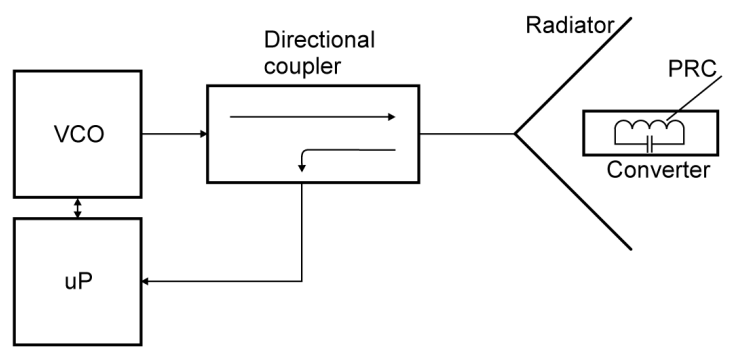

Fig.6. Flow chart of the sensor.

The microcomputer can measure the frequency of the reflected wave from the transfer characteristic of the VCO, or it can be measured directly by means of sum of transitions of the transmitted signal with zero value for a unit of time. Each method has their advantages and disadvantages. Due to minimization of the influence of the nonlinear transfer characteristic of the VCO on the accuracy of measurement, the second possibility has been used in our case.

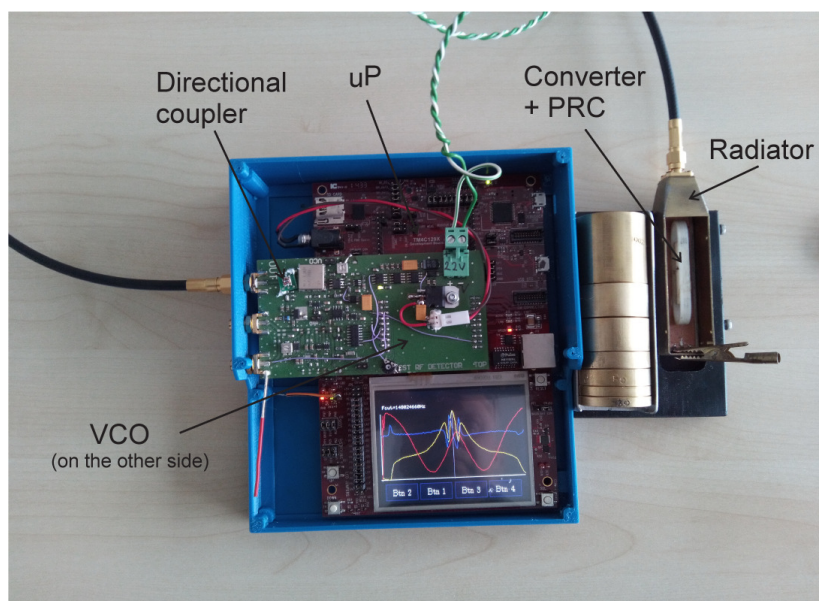

Fig.7. Realization of a one-component sensor of force.

The block diagram of the force sensor provided the basis for the fabrication of the hardware. So far only a laboratory version of the electronic accessory of the MEMS sensor of force has been constructed. The base of it is comprised of the commercially available and sufficiently powerful 
TM4C129X microcomputer development kit (represented as uP in Fig.6.). To this kit a printed circuit board was fabricated which contained a voltage controlled oscillator with transformer directional coupler and several highfrequency detectors (for optimum performance tuning). This device supplies the TEMCell, and at the same time it scans the reflected wave and evaluates the frequency of the reflected wave down to units of hertz. The device in its operational state is shown in Fig.7. as it measures the reflected wave from the unloaded elastic element with frequency around $140 \mathrm{MHz}$.

After loading of the flexible element the capacity of the capacitor (5) will increase and the displayed frequency will drop (9). The principle and the block diagram have been used in the industrial design [12].

This electronic equipment makes it possible to scan the reflected wave from the elastic element in a wide frequency range that is largely due to properties of the voltage controlled generator and the ability of the microcomputer to measure the maximum frequency. This setup permits to measure the transfer characteristic of the elastic element fabricated from various elastic materials with dimensions in the order of centimeters. At the same time it allows calibration and adjustment. Due to the fact that the frequency of the reflected wave is measured with accuracy of several hertz, loss of information about the measured quantity does not occur (physical quantity of time or, in this case of frequency, is measurable more accurately comparing to the quantity of weight or force, respectively). Moreover, as the elastic element is fixed onto a hard (steady) bearing and the signal carrying information about the physical quantity is transferred wirelessly, the influence of the environment to the accuracy of the measurement of the physical quantity is minimized.

\section{RESUlts}

Based on chapter 2.3., an elastic element was printed from polyamide (PA) material PA6/66 using a 3D printer. Compact compliant mechanical body (CCMB) shape is shown in Fig.8. and its dimensions are displayed in Table 1. From individual dimensions of the CCMB we may determine the starting values both for the capacitor and the inductor. The sensor in Fig.7. scans the reflected wave and measures/calculates its frequency which depends on the PRC being part of the CCMB and thus forming the MEMS. Specifically, this frequency depends on the elements which form the PRC, that is on the coil $L$ and the capacitor $C$. With respect to Fig.8. and Table 1., the capacity of the capacitor will be

$$
C=\varepsilon_{0} \varepsilon_{r} \frac{S}{d}=8.86 \cdot 10^{-12} \frac{0.037 \cdot 0.0056}{0.00175-k F}[F]
$$

where $\mathrm{F}$ is the acting force and $\mathrm{k}$ is the corresponding stiffness constant of the CCMB. For the inductance of an oval coil a rather complicated equation is in force that depends again on the dimensions of the CCMB. However, first we have to calculate the MEMS area

$$
\begin{aligned}
S_{L} & =\pi \frac{h_{L C}}{2} \frac{l_{L C}-a_{L C}}{2}+a_{L C} h_{L C} \\
& =\pi \frac{10.5}{2} \frac{52.0-37.0}{2}+37.0 \cdot 10.5=512.2\left[\mathrm{~mm}^{2}\right]
\end{aligned}
$$

And based on [16] we will obtain

$$
L=\frac{13 \cdot 10^{-6} N_{L}^{2} S_{L}}{w_{L C}} 10^{6}=\frac{13 \cdot 3^{2} \cdot 512.2}{37}=1070[\mathrm{nH}]
$$

While the shape of a MEMS changes by acting force $\mathrm{F}$, the coil slightly $(\approx \%$ ) ) changes its inductance what is also included into that calculation. Experimentally it has been found that

$$
L=1070 \cdot 10^{-9}-1.566 \cdot 10^{-9} \cdot F[H]
$$

Then the resonance frequency of a parallel resonance circuit placed at the surface of a CCMB will be

$$
f=\frac{1}{2 \pi} \frac{1}{\sqrt{L \cdot C}}
$$

Taking into consideration the character of equations (9) and (5) we may expect a non-linear transfer characteristic of a one-component force. This can be verified by using a decoding microcomputer with the help of which we can make a conversion table which will consist of calibrating constants for individual points of the incident quantity. The values between individual calibrated points can be replaced by a line (straight line interpolation). The more points this table contains, the less interpolation errors will occur.

Table 1. Compact compliant mechanical body dimensions.

\begin{tabular}{|l|c|c|}
\hline Dimension & Design & Real \\
\hline$l_{L C}[\mathrm{~mm}]$ & 50 & 52.0 \\
\hline$h_{L C}[\mathrm{~mm}]$ & 11 & 10.5 \\
\hline$w_{L C}[\mathrm{~mm}]$ & 5 & 5.6 \\
\hline$a_{L C}[\mathrm{~mm}]$ & 30 & 37.0 \\
\hline$d_{L C}[\mathrm{~mm}]$ & 2 & 1.75 \\
\hline$N_{L}[-]$ & 3 & 3 \\
\hline
\end{tabular}

To verify the method described herein, the static conversion characteristics of the CCMB were measured by using the device described in chapter 3. In the TEMCell a MEMS was placed, to which using a piston acted the weight of the load. The piston was designed so that it worked upon a small area right in the center of the elastic element. The output variable was frequency that was directly displayed by a one-component force sensor. The advantage of this method is an easy replacement of the MEMS and relatively fast measuring of its transfer characteristics. On the other hand, the disadvantage is a friction of the piston with its body in which it moves or, a possibility of it getting stuck. 


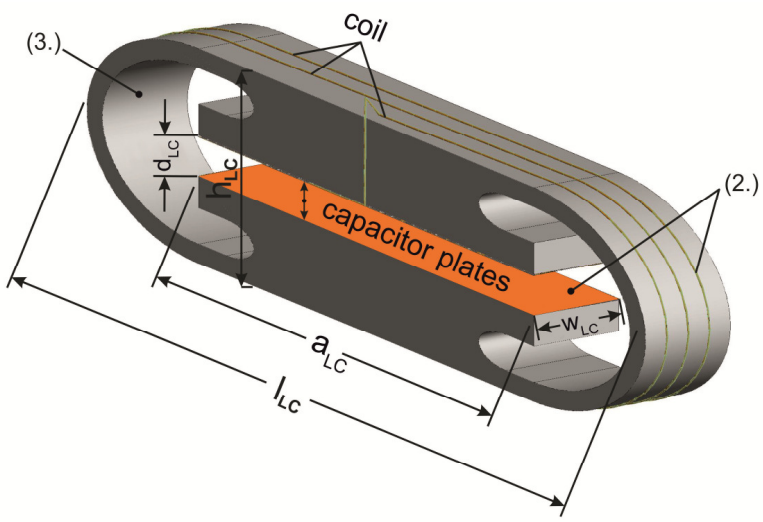

Fig.8. Compact compliant mechanical body.

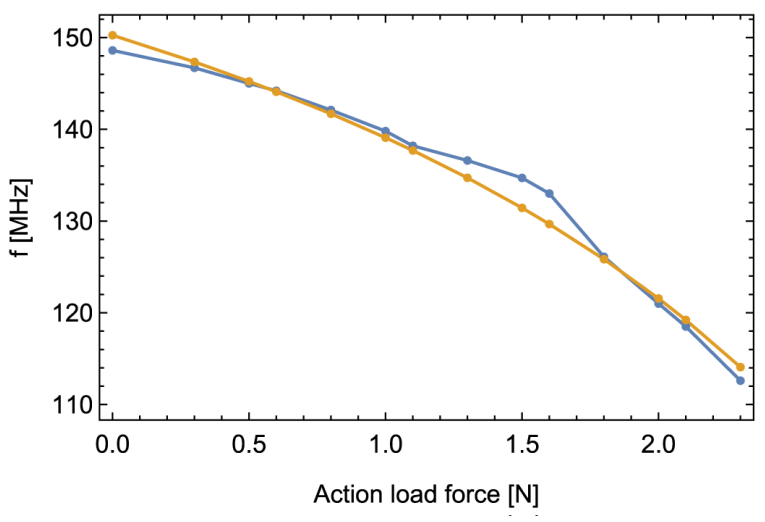

Fig.9. Transmission characteristic of the proposed compact compliant mechanical body, (yellow - theory, blue - measure by the principle on Chapter 3.)

In Fig.9. theoretically calculated transfer characteristic of an elastic element (light orange) and its measured characteristic (light blue) is shown. Both curves are markedly similar and their shape follows the same function. At the beginning and at the end of the measured characteristic there is a deviation from the theoretically calculated curve. This is very likely due to imperfect defining of the real dimensions of the $\mathrm{CCMB}$, in particular the distance $\mathrm{d}_{\mathrm{LC}}[\mathrm{mm}]$. At load of $\approx 1.5 \mathrm{~N}$ a significant shift of the characteristics occurs. This was already the case of imperfect piston pressure on the CCMB caused by bad mechanical conversion of weight to force.

Despite the variations in the curves in the level of some per cents, the novel physical measurement method of measuring physical quantities and contactless data transmission has been successfully verified.

\section{CONCLUSIONS}

The article aims to familiarize readers with a novel method of measuring physical quantities with conversion to frequency. The described method is based on changes in EPM properties in which the sensor of physical quantity is located. It is a remote type of resonance method with the difference in that the information on the acting mechanical quantity is transmitted wirelessly, which increases the accuracy of the measurement. A MEMS sensor was used, which compared to commonly available sensors (e.g., thermometric) has significantly better static features. Excellent dynamic characteristics can be assumed which, however, depend on the natural frequency of the CCMB and on the ability of electronic equipment to capture rapid changes. Finding other dynamic and static parameters of CCMB will be the subject of further research.

\section{ACKNOWLEDGMENT}

This work was supported by the national scientific grant agency VEGA under the project No.: 2/0155/19 "Processing sensoric data via Artificial Intelligence methods", projects APVV 14-0076 "MEMS structures based on load cell", APVV 15-00624 "Electromagnetic compatibility ensuring of monitoring systems of abnormal operating condition of nuclear power plant". The authors also thank to the support within the frame of operating program Research and Development for the Project: "Research of the technological basis for design of applications utilizing the renewable sources of energy in practice", with ITMS code: 26220220083, co-financed by resources of European Regional Development Fund. The final thanks for the support go to the "Joint Research Project" SAS-MOST JRP 2017/1.

\section{REFERENCES}

[1] Nie, B., Yao, T., Zhang, Y., Liu, J., Chen, X. (2018). A droplet-based passive force sensor for remote tactile sensing applications. Applied Physics Letters, 112, 031904. https://doi.org/10.1063/1.5005873.

[2] Nakazato, Y., Kawanaka, K., Takita, K., Higuchi, M. (2017). Development of peristaltically propelled active catheter used in radial artery. In Microactuators and Micromechanisms: Proceedings of MAMM-2016, Ilmenau, Germany, October 5-7, 2016. Springer, 7990. https://doi.org/10.1007/978-3-319-45387-3_8.

[3] Howell, L.L., Magleby, S.P., Olsen, B.M. (eds.) (2013). Handbook of Compliant Mechanisms. John Wiley \& Sons. https://doi.org/10.1002/ 9781118516485.ch1.

[4] Wei, J., Fatikow, S., Zhang, X., Haenssler, O.C. (2018). Design and experimental evaluation of a compliant mechanism-based stepping-motion actuator with multi-mode. Smart Materials and Structures, 27, 105014. https://doi.org/10.1088/1361-665X/aad79e.

[5] Kumar, K., Zindani, D., Kumari, N., Davim, J.P. (eds.) (2019). Micro and Nano Machining of Engineering Materials. Springer International Publishing. https://doi.org/10.1007/978-3-319-99900-5.

[6] Lu, H., Wang, P., Tan, R., Yang, X., Shen, Y. (2018). Nanorobotic system for precise in situ threedimensional manufacture of helical microstructures. IEEE Robotics and Automation Letters, 3 (4), 28462853. https://doi.org/10.1109/LRA.2018.2846051.

[7] Plander, I., Stepanovsky, M. (2018). Interdisciplinary considerations on the design of MEMS actuators from a perspective of their optimality. Sensors and 
Actuators A: Physical, 269, 203-211. https://doi.org/10.1016/j.sna.2017.11.007.

[8] Hricko, J., Havlik, S. (2017). Flexural body for a wireless force/displacement sensor. Microactuators and Micromechanisms: Proceedings of MAMM-2016, Ilmenau, Germany, October 5-7, 2016. Springer, 5966. https://doi.org/10.1007/978-3-319-45387-3 6.

[9] Hartansky, R., Hallon, J. (2016). Sensors for Measure Electromagnetic Field [Senzory pre meranie elektromagnetickeho pola]. Bratislava: Slovenska technicka univerzita v Bratislave, ISBN 978-80-2274565-9. (in Slovak)

[10] Hartansky, R., Marsalka, L. (2012). Wire structures mutual impedance change in electromagnetic field. Journal of Electrical Engineering, 63 (7s), 152-155.

[11] Marsalka, L., Hartansky, R. (2011). Electromagnetic method for distance measurement on MEMS structures. In MM Science Journal: Proceedings of the RAAD 2011, 20th International Workshop on Robotics in Alpe-Adria-Danube Region (RAAD), 5-7 October 2011, Brno, Czech Republic, Special Edition, 48-53.

[12] Hartansky, R., Halgos, J., Hricko, J., Rafaj, M. (2019). Method and device for contactless sensing of mechanical quantities [Sposob a zariadenie na bezkontaktne snimanie mechanickych velicin]. Slovak Utility Model SK 8653 Y1. (in Slovak)

[13] Muller, I., de Brito, R.M., Pereira, C.E., Brusamarello, V. (2010). Load cells in force sensing analysis -theory and a novel application. IEEE Instrumentation
\& Measurement Magazine, 13 (1), 15-19. https://doi.org/10.1109/MIM.2010.5399212.

[14] Liang, Q., Wu, W., Zhang, D., Wei, B., Sun, W., Wang, Y., Ge, Y. (2015). Design and analysis of a micromechanical three-component force sensor for characterizing and quantifying surface roughness. Measurement Science Review, 15 (5), 248-255. https://doi.org/10.1515/msr-2015-0034.

[15] Allan, H.R., Curling, C.D. (1948). The design and use of resonant cavity wavemeters for spectrum measurements of pulsed transmitters at wavelengths near $10 \mathrm{~cm}$. Journal of the Institution of Electrical Engineers - Part III: Radio and Communication Engineering, 95 (38), 473-484. https://doi.org/ 10.1049/ji-3-2.1948.0117.

[16] Books, M., Turner, H.M. (1912). Inductance of coil. University of Illinois Bulletin, 9 (10), 53.

[17] Mierka, M. (2019). Realization of a TEM cell. In Measurement 2019: 12th International Conference on Measurement. Bratislava: Slovak Academy of Sciences, 162-166. https://doi.org/10.23919/ MEASUREMENT47340.2019.8779907.

Received April 25, 2020 Accepted July 13, 2020 